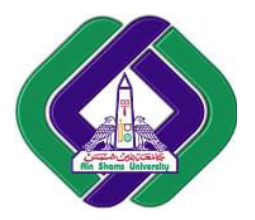

International Journal of Intelligent Computing and Information Sciences

\title{
Perfromance Analysis of aTree Routing Protocol for Cognitive Radio Network Under Impact of Different Primary User Activity Patterns.
}

\author{
M. Hashem \\ Department of information Systems, \\ Faculty of Computers and Information Sciences, \\ Ain Shams University, \\ Cairo, Egypt \\ mhashem100@yahoo.com
}

\author{
S. I. Barakat \\ M. A. AttaAlla \\ Department of information Systems, \\ Faculty of Computers and Information Sciences, \\ Mansoura University, \\ Mansoura ,Egypt \\ shriefiib@yahoo.com m3ttaalla@gmail.com
}

\begin{abstract}
The mistakes in the spectrum management have drive to a problem of the scarcity of spectrum, which can be solved through the use of Cognitive Radio (CR) technology. The main concept of CR technology allows unauthorized users (secondary users (SUs))for using the spectrum resources for communication without harm authorized users (primary users (PUs)).The traditional wireless networks, the nodes are connected based on a centralized infrastructure. But in Cognitive Radio Ad Hoc Network (CRAHN) Networks, mobile SUs nodes are connected to each other without a central infrastructure. Routing in CRAHNs is an important and necessary process in CRAHNs. It faces many and various challenges. These challenges include PUs intervention, frequently dynamic topology, energy consumption problems, unavailable channels, and fragile connections between nodes. The our probased routing protocol called A tree routing protocol for cognitive radio network (C-TRP) has utilized the tree routing algorithm with a spectrum management module in one routing decision module. In this paper, weevalute a performance of the tree routing protocol $(C-T R P)$ under the influence of the different PUs activity patterns with help of three performance metrics :hop count, the number of CR nodes and number of available channels. The performance evaluation is evaluted using NS-2 simulator. Simulation results confirm that C-TRP outperforms "spectrum-tree based on demand routing protocol for multi-hop cognitive radio networks"(STOD-RP), and Cognitive Tree-based Routing $(C T B R)$ in terms of packet delivery ratio and causes less harmful interference to $P R$ nodes, and also less end-to-end delay in all primary radio nodes activity patterns.
\end{abstract}

Keywowords:Multi-hop cognitive radio networks, Cognitive radio ad hoc network, spectrum diversity, performance evaluation, CRAHNs.

\section{Introduction}

Due to the ever-increasing demand for mobile devices and the continuous development of wireless technology, frequency spectrum has become a costly and scarce resource. Furthermore, the problem of the unutilized spectrum resulting from current fixed spectrum distribution policies. These policies allow licensed wireless devices only to communicate over fixed spectrum band [1]. The result according to a report [2] by the "Federal Communications Commission (FCC)" (FCC's Spectrum Policy Task Force 2002) is a large of the spectrum licensed under current spectrum policies is used sporadically, ranging from $15 \%$ to $80 \%$ as described in Figure 1. 
Figure 1: The usage of licensed spectrum.

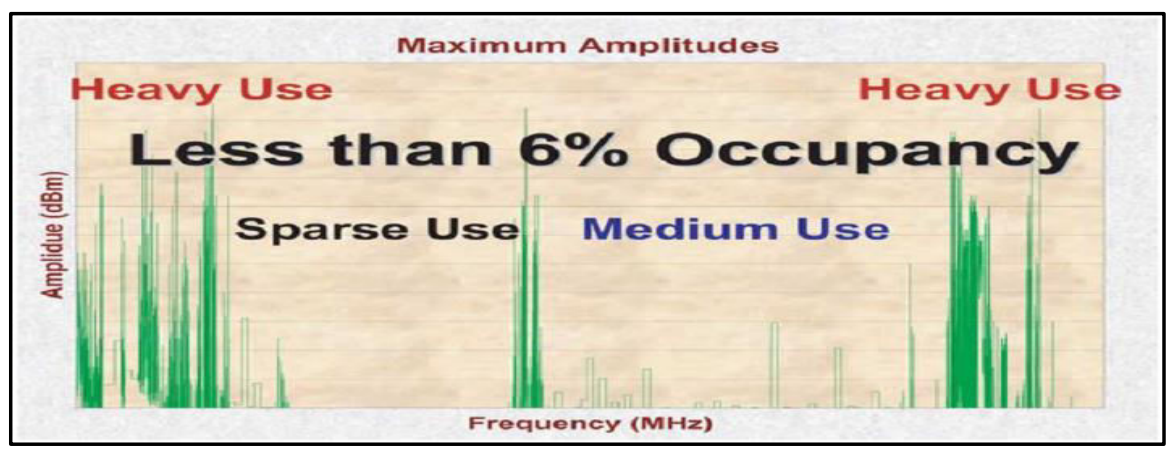

The report finds that large parts of the licensed spectrum are not exploited and are still available for usage [3]. These time and places are called spectrum opportunities (SOP). CR is an innovative solution $[3,4,5]$ for optimizing those SOPs and solving these problems of spectrum scarcity. The CR is enable unauthorized users (SUs) to exploit licensed spectrum bands to use it in a smart and careful manner without any harmful interference to the authorized users (PUs). CRAHNs [6] is a type of mobile ad hoc network and has the characteristics of cognitive radio networks (CRNs). In CRAHNs, SUs collaborate with each other in a distributed manner without the using of the centralized infrastructure. Each node takes decisions and changes its parameters through the help of the software define radio (SDN) technology [7]. The CR node can monitor the status of the existing spectrum and make are appropriate decisions according to the current situation and then take actions (such as changing transmitter parameters or switching the transmission channel). The available SOPs in wireless networks areconsistingfrom several channels (not a single channel). SUs can temporarily exploit theseSOPsthat currently are not used by PUs. Once the SUs discover the appearance of PUs on these channels, the SUs must vacate immediately these channels.

Many researches in the CRAHNs are related to the lower layers, which include the MAC layer and the physical layer, which aims to develop a new innovative techniques in spectrumsensingmodule,spectrum-sharing module and spectrum-decision module, however the subject of routing is considered one of the most critical subjects that requires further studies and research to be discovered and implemented efficiently and effectively $[8,9]$.In traditional wireless networks, each node has a fixed available channel for communication, but in CRAHNs, the situation is different because each node has a various channels for communication and not available all the time because the mobility of the nodes. In addition, the available channels may change based on PUs' behavior. The mobility and the PUs' behavior in CRAHNs have a negative impact on connections between nodes and should be taken into consideration when designing an effective routing protocol for these networks.

In this paper,weanalyze and study the effect of various PUs activity patterns on various tree routing protocols i.e. STOD-RP [10], CTBR [11] and C-TRP [12]. Furthermore, in our protocol proposed will also analyze the different responses of these protocols to the different PUs activity patterns. Performance is assessed through simulations NS-2 simulator tool.In this paper, the results obtained from the NS-2 simulator using a visual trace analyze tool [13].

The rest of this paper is organized as follows: section 2 Give a brief overview of routing protocols for CRN and related works. In section 3, we will explain our tree routing protocol for cognitive radio 
network (C-TRP). In the 4 section, the different patterns of PU activity will be explained. In Section 5 , the performance is evaluated .Finally, the conclusion in Section 6.

\section{Related Work}

The stability of routes in the CRAHNs is highly depending on the pattern of PU activity. The PUs activity pattern, i.e. channel busy / free can be modelled as continuous-time, alternating BUSY/FREE Markov Renewal Process (MRP). This model was used to model PUs activity in these previous studies $[14,15,16]$.

Recently, there is little work to analyze the activity of PUs on the channels. In this research [17], the performance of TCP (transmission control protocol) protocol was analyzed and evaluated over cognitive Ad doc networks. The authors considered a single hop network topology and four types of PUs activity ((prolonged-range, high-range, soft-range, and sporadic-range) were analyzed. In [18] the performance of MAC protocol was assessed under the influence of the different occupancy / availability periods of the PUs activity. The authors [19] studied the effect of the PUs behavior on the area in which SUs have opportunities for spectrum reuse, with the transmission capacity given. However, none of these works have analyzed and studied the effect of different PUs activity patterns on the routing protocols. Moreover, none of these works, which studies and analyzes the effect of PUs activities in the CRAHNs environment. In fact, the task is difficult in the CRAHNs networks because there is no central infrastructure and there are no permanent channels available between SUs' nodes.

In this paper considers three routing protocols for the CRAHNs i.e. STOD-RP [10], CTBR [11] and our proposed routing Protocol C-TRP [12]. In STOD-RP protocol tackles the problem of integration between the routing unit and the channel selection management unit. The STOD-RP selects a node from the existing nodes in the topology to become the root node of the tree and also constructs one separate tree for each spectrum band. Each node sends CR node information such as the available channels to the root node that operates as a central infrastructure. The root node is determined based on the node with the largest number of spectrum bands and has the longest period of spectrum availability. The STOD-RP selects the route based on both statistical PUs activities and SU Quality of Service (QoS) requirements. The STOD-RP's Drawbacks: First run the proactive routing tree to get routes from the node to the root node, and then use on demand discovery to search the destination node, which may cause overhead handling and longer delay. The second disadvantage is that the STOD-RP protocol that uses the only channel available in the tree to send control packets and does not use a common control channel between the nodes, which reduces the network throughput and increases the delay from end to end. Furthermore, it affects the stability of the topology of the tree because the channel is not always available due to PU activities.

Another routing protocol that uses the tree algorithm method in the design of a routing protocol for CRAHNs is "cognitive tree based routing (CTBR)". A CTBR has enhanced tree based routing to adapt multiple wireless systems. The CTBR protocol is an extension of the original TBR protocol with some modifications to adapt to CRNs. The authors assumed that a central infrastructure unit would work as a root point for the tree. The access point (root node) floods a periodic message to all nodes called a root announcement (RANN) messages. When the node receives a RANN message, the node retains the parent node that sent this message as a potential parent. Then, each node selects only 
one parent from the potential parents based on the best path cost function from this node to the root node. When the node receives the RANN message, it replies to the upper direction to the root node by another message called the root reply (RREP) message so that the nodes register themselves at the root node. In the end, the root node records all the nodes which responded with a REP message in its routing table and thus, a tree is built from nodes and the root node can reach to all the nodes. The authors proposed two routing metric methods for select the parent node. The first method is called cognitive-aware link cost function for a global decision schema from end to end route, Whereas the second one called local decision method, which selects the best interface for sending the packets, based on least load interface. The disadvantage of CTBR is that it depends on the root node, which is basically access point and this assumption is unacceptable in the CRAHNs because the CRAHNs environment does not have a central infrastructure.

\section{The Tree Routing Protocol For Cognitive Radio Ad hoc Network (C-TRP)}

In a previous study [12], we proposed tree routing protocol for CRAHNs (C-TRP). In the C-TRP protocol, the tree begins to form by sending messages called the root advertisement (RA). The message is periodically broadcasted across all available channels. The RA message contains the field called the sequence number that is increased by one at each transmission. Any node in theology receives the RAmessage, it stores the node to which the message was sent as a possible parent and then rebroadcasts the message to its other neighbors after updating another field called the cost filed. After waiting for arrival of another RA messages from the other channels, the node identifies the best parent. The best parent node is the node with best weight value.Each node selects a definite path to the root node.Each node receives the RA message reply with a new message called a Node Acknowledgment (NA) and sends the NA message to the root node. Each intermediate node that receives a NA message sends it forward in the direction of the parent node via its best path and immediately updates its routing table by recording the node which is NA message source. Finally, the root node identifies all nodes and builds a tree to access any node in the tree topology.

The C-TRP protocol computes its calculations and chooses a single root node, consequently the other nodes discover all paths to root node. When there is more than one channel at the node, the C-TRP algorithm chooses the best channel based on the weight equation as explained it in the next section. The best channel can be used to exchange data packets. In addition to selecting the best channel, the C-TRP also disables the other channels to avoid interference and achieve a fair spectrum distribution paving the way to other nodes to explore the free channels.

C-TRP uses our previous algorithm to select the best available channels from one node to another, this algorithm is called "Distributed Channel Selection Strategy Based on Channel Weight (DCSS)" [20]. C-TRP arranges the available channels from best to lowest based on an equation called the channel weight equation. The channel weight equation in C-TRP includes two parameters, first is the PU unoccupancy probability and second parameter is the channel delay. The PU unoccupancy probability is the possibility that the PUs nodes reserves the channel, C-TRP prefers selecting the least probability occupied channel by PUs. The second parameter is delay because we need transfer data rapidly. So, each SUs' node running C-TRP, locally computes using the following equation:

$$
\mathrm{C}_{\mathrm{w}}^{\mathrm{i}}=\mathrm{PR}_{\mathrm{u}}^{\mathrm{i}} \times 1 / \text { delay }
$$


$\left(C_{w}^{i}\right)$ describes the weight of a channel (i) and $P R_{u}^{i}$ is the PU unoccupancy and delay is the delay over channel i.C-TRP will arrange the channels according to the highest weight based on the equation (1). The highest priority for the C-TRP is to protect the PUs, so the probability that PUs are idle on channel is preferred, i.e.PR $R_{u}^{i}$. The second goal of C-TRP is to use the delay as another criteria in equation (1) when the delay increases, the weight of the channel decreases and vice versa. In the following section, the estimation of the PUs unoccupancy and channel delaywill beclarified.

The PUs' signal on a channel can be modelled as continuous alternating Free/Busy Markov Renewal Process [14]. Whereas the busy time is the time that the channel is occupied by PUs' traffic, The Free time is the time when channel is available to CR node for sending data packets. Since each channel's status (i.e. Free/Busy) is independent, each status alternates from free to busy or from busy to free according to the Poisson arrival process. The time periods of the channel i statuscan expressas follows: $T_{\text {free }}^{i}$ and $T_{\text {busy }}^{i}$. Where $T_{\text {free }}^{i}$ represents the period in which the channel is available to SUs and $T_{\text {busy }}^{i}$ Represents the period in which the channel is reserved by PU. The periods in which the channels are free or busy are supposed to be independent and distributed $[14,15,16]$. Because the appearance of PU on channels is independent of other users, so every change in PU's activity follows Poisson's access. In our paper [20] calculate $P R_{u}^{i}$ is calculated as shown in equation (2) that equals time the channel be available. More details on how to calculate these possibilities can be found in our paper.

$$
\mathrm{PR}_{\mathrm{u}}^{\mathrm{i}}(\mathrm{t})=\lambda_{\mathrm{x}} / \lambda_{\mathrm{x}}+\lambda_{\mathrm{y}}+\lambda_{\mathrm{y}} / \lambda_{\mathrm{x}}+\lambda_{\mathrm{y}} \mathrm{e}^{-\left(\lambda_{\mathrm{x}}+\lambda_{\mathrm{y}}\right) \mathrm{t}}
$$

The second parameter is delay at each node which can be calculated by using two delay types: channels switching delay $\left(\right.$ Chdelay $\left._{s w}\right)$ and channel queuing delay $\left(\right.$ Chdelay $\left._{q}\right)$ as show in the equations (3).

$$
\text { Delay }_{\text {node }}=\text { Chdelay }_{\text {sw }}+\text { Chdelay }_{\mathrm{q}}
$$

It was explained how to calculate the node delay value, which includes both the switching delay and the queuing delay in detail in the previous paper [20].

\section{PU's Behavior Pattern}

Definingthe periods of PUs activity on the available channels are not predictable, but we can make a model that simulates this activity. PU activity on the channel can be represented in one of two cases busy /free; in the case of the busy situation there is an activity of PUs on the channel and in this case the channel is not available to the SUs. In free case in the sense that the channel is free of any activities of PU on it and can SU utilizes the channel. PU state, can be modelled as continuous-time, alternating busy/free "Markov Renewal Process (MRP)" [14, 15, 16]. In this paper, the equation in $[14,15,16]$ is used which period of busy and free are distributed exponentially $g_{x(t)=\lambda_{x} * e^{-\lambda x t}}$ for busy state and $g_{y(t)=\lambda_{y} * e^{-\lambda y t}}$ for Free State. We can calculate the probability that the channel is free 
PUs activity by using equation (2) where $\lambda_{x}$ and $\lambda_{y}$ are the rate parameter for exponential distribution.

The four different patterns of PUs activity are $[17,18]$ as shown in Figure 2:

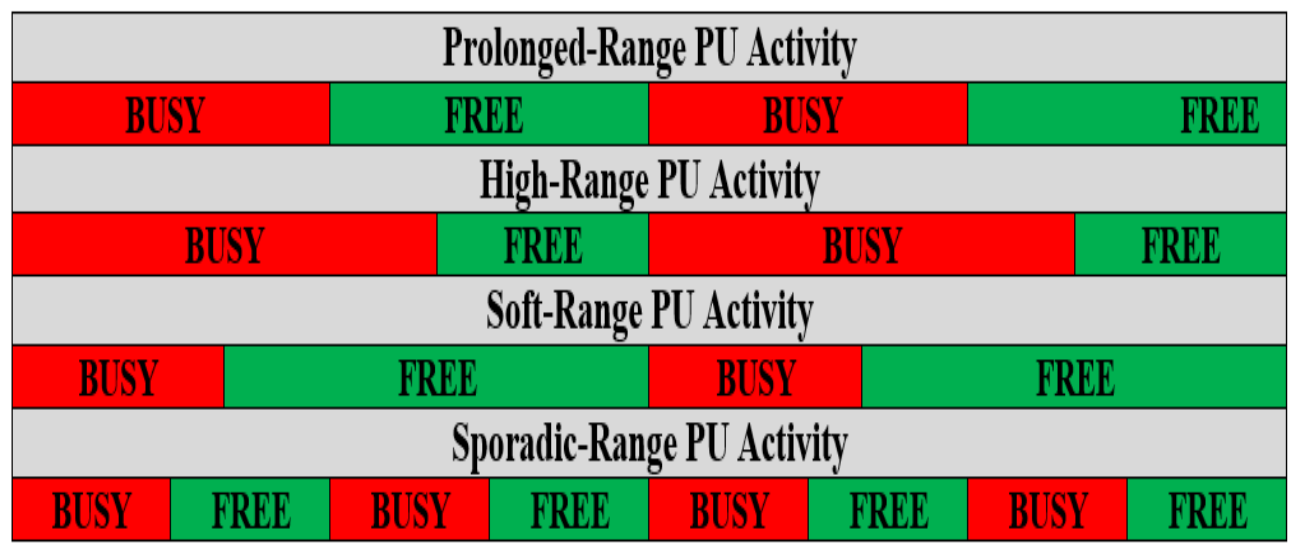

Figure 2: The PU behaviour patterns

1. Prolonged-Range: In this mode, the channel has a long busy time and a long free time. This mode of PU activity can occur in cases where the PU's node is subscribed in free call packets for its cellular networks.

2. High-range: In this type of PU's activity, the channel has a long busy period and in contrast, a short period of free time. This type can be seen at peak time or densely populated cities, where all channels are mostly reserved.

3. Soft-range: in the soft range, the channel has short busy periods and long free times. This pattern of PU behavior can be noted in remote areas or during less peak night hours.

4. Sporadic-Range: in the sporadic-range, the channel is busy for short periods and is also free for a short periods. This pattern of PU's activity can be noted as users use frequency bands for a very short period of time, such as using networks available in public transportation, trains, etc.

The patterns of the previous four activities are shown in Figure 2. In order to obtain the values for the four activities, we have changed values $\lambda_{x}$ and $\lambda_{y}$ as shown in Table (1) $[17,18]$.

Table 1:PU's Activities pattern

\begin{tabular}{|c|c|c|c|c|}
\hline Pattern & Busy & Free & $\lambda_{x}$ & $\lambda_{y}$ \\
\hline Prolonged & $\lambda_{x} \leq 1$ & $\lambda_{y} \leq 1$ & Long Busy & Long Free \\
\hline High & $\lambda_{x} \leq 1$ & $\lambda_{y}>1$ & Long Busy & Short Free \\
\hline Soft & $\lambda_{x}>1$ & $\lambda_{y} \leq 1$ & Short Busy & Long Free \\
\hline Sporadic & $\lambda_{x}>1$ & $\lambda_{y}>1$ & Short Busy & Short Busy \\
\hline
\end{tabular}




\section{IJICIS, Vol.17 No. 4 october 2017}

\section{PerformanceEvaluations and Results}

\subsection{System model}

In this section, we study the performance of the three routing protocols C-TRP, STOD-RP, and CTBR under the influence of the four previous types of PU's activities. For the performance study, using an extensive NS-2 simulation based on three measures of performance metrics:

1. Harmful interference ratio (HIR): It is defined as a proportion of the total number of times that the channel is reserved to the PU's node after the channel is selected for SU's nodeover the total number of times that this channel was selected by SUs.

2. Packet Delivery Ratio (PDR) is the rate of data received at the destination nodes to data sent from the source nodes.

3. The average end-to-end delay is defined as the average time that packets of data need to be delivered over a network from one source to destination.

The Visual Tracking Analyser tool [13] will be used with a NS-2 program to get simulation output trace files. The simulation results of average HIR, PDR, average end-to-end delay are shown in the form of line graphs. In this experiment, the number of SU's nodes are fixed at 100 nodes. SU's nodes are distributed randomly within a square area of $1000 \times 1000 \mathrm{~m} 2$ area and the data transmission range is set to $250 \mathrm{M}$. The experiment will be run for 1000 seconds and 1000 packets will be sent in each experiment at a rate one packet per second and the source node will be chosen randomly. A 95\% confidence interval has been applied to all results. The evaluation of C-TRP performance by comparing it with two routing protocols, STOD-RP and CTBR, we comparing it with STOD-RP and CTBR as, they are tree routing protocol. The performance is evaluated under the different PU pattern activities.

\subsection{Results}

\subsubsection{Harmful interference ratio (HIR)}

In this section, we will evaluate the effect of HIR on PU by SUs for testing under a variable number of channels. We mean, in other words, the interference caused by SUs nodes transmissions to the PUs nodes of C-TRP, CTBR, and STOD-RP. Figure 3(a-d) evaluate the HIR for the three routing protocols i.e. CTBR, STOD-RP and C-TRP with different number of channels $(\mathrm{Ch} .=5$ and $\mathrm{Ch} .=10)$. It can be shown in the figure 3 shows as expected that C-TRP outperforms the other two's protocol and causes less HIR to the PUs'. This is primarily because, when using C-TRP, SUs nodes select those channels that have high probability that PUsare not exist or in another meaning in free state, Thus reducing interference with the PUs nodes. 


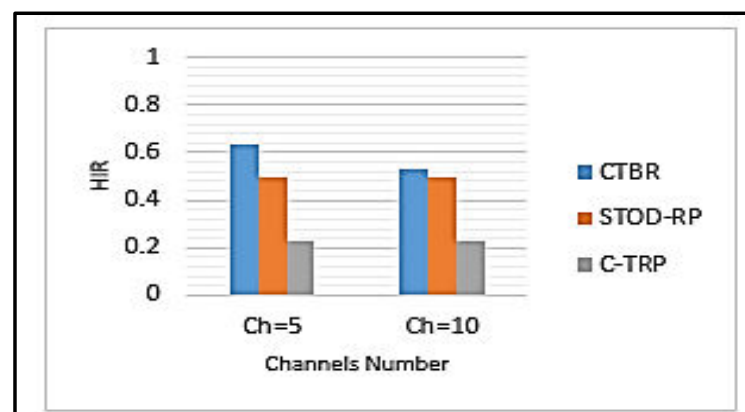

(a) HIR under Prolonged-range of PU activity to CTBR, STOD-RP-C-TRP

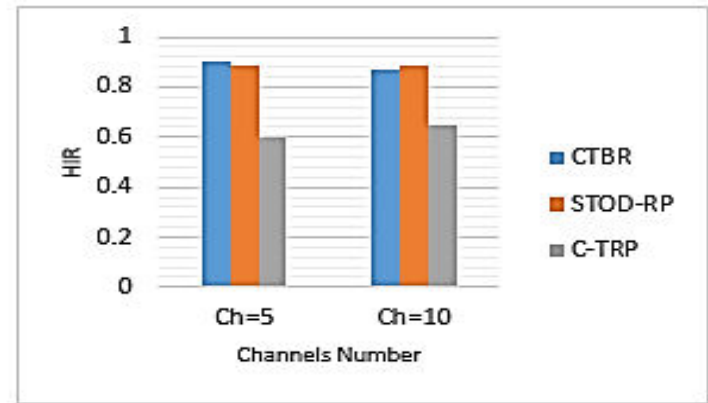

(c) HIR under High-range of PU activity to CTBR, STOD-RP-C-TRP

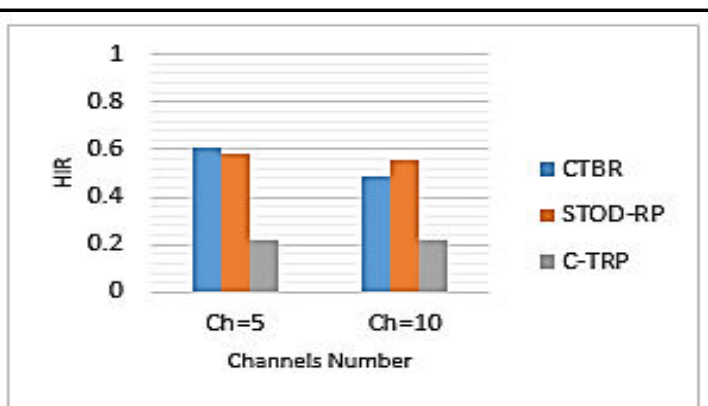

(b) HIR under Sporadic-range of PU activity to CTBR, STOD-RP-C-TRP

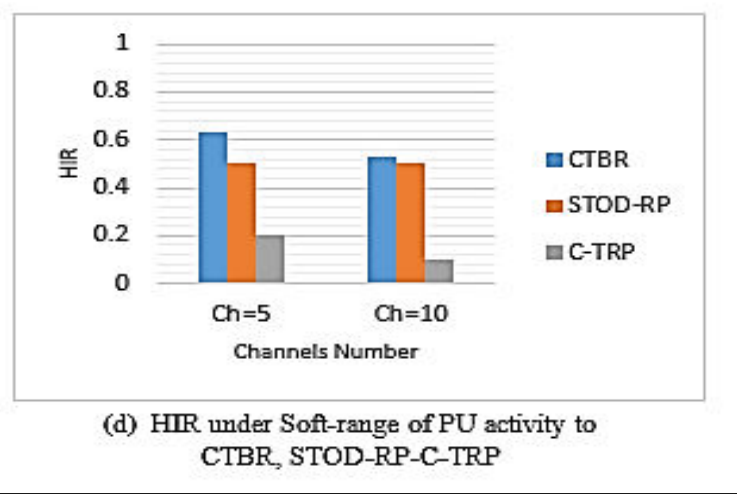

Figure 3 Comparison of HIR among CTBR, STOD-RP, C-TRP Under impact of different PU Activity patterns and different Number of Channels

The higher HIR value for C-TRP shown in Figure 3 (c) that reflects cases where all channels were not available and are occupied by the PUs nodes and potential interference may occur in the case of packet transmission. So we prefer if all the channels in busy state, stop the transmission process. Moreover, when the number of available channels decreases from 10 to 5, the probability that the PUs nodes will find channels in a free state to transfer their data will be decreased. As a result, CTRP protects the PUs nodes, by minimizing the amount of interference with the PU nodes.

In a High-Range PUs activity, the whole channels is highly busy, meaning $P_{O F F}(t)$ is small that indicates that the window time available for transmission will be shorter and the transmission of SUs are more likely to be interrupted by the long presence of the PUs and consequently, very less chances for communication is let to all the approaches to interference with PUs nodes However, C-TRP is capable of managing very low HIR compared to other two approaches and HIR rate is improved in a Sporadic-Range and Prolonged-Range patterns than High-Range state because the $P_{O F F}(t)$ increase. It is clear that when PUs activities were very low as shown in figure 3(d) every strategy behaves well in term of HIR.C-TRP generates very less HIR, when compared to CTBR, STOD-RP.

\subsubsection{Packet Delivery Ratio (PDR)}

A higher percentage of PDR is required in any network. Weanalyses the performance of the CTBR, STOD-RP and C-TRP by assessing the ratio of PDR. Figure 4 (a-d) compares the ratio of packets delivery of CTBR, STOD-RP and C-TRP under different two parameters first, changing thenumber 
IJICIS, Vol.17 No. 4 october 2017

of the channelsCh. $=5$ to $\mathrm{Ch} .=10$, the second one is the changing number of SUs nodes from 10 nodes to 35 nodes. Figures 4 (A-D) show that C-TRP has a higher ratio when compared to a CTBR and STOD-RP. This is due to the fact that C-TRP depends on the PUs activity, while choosing a channel to transfer data. Moreover, in C-TRP, the ratio of the lost packet decreases, when the number of channels increases from $\mathrm{Ch} .=5$ to $\mathrm{Ch} .=10$. This is primarily because when the number of channels increases, C-TRP has higher chances for finding channels in Free State and using it for transmission.

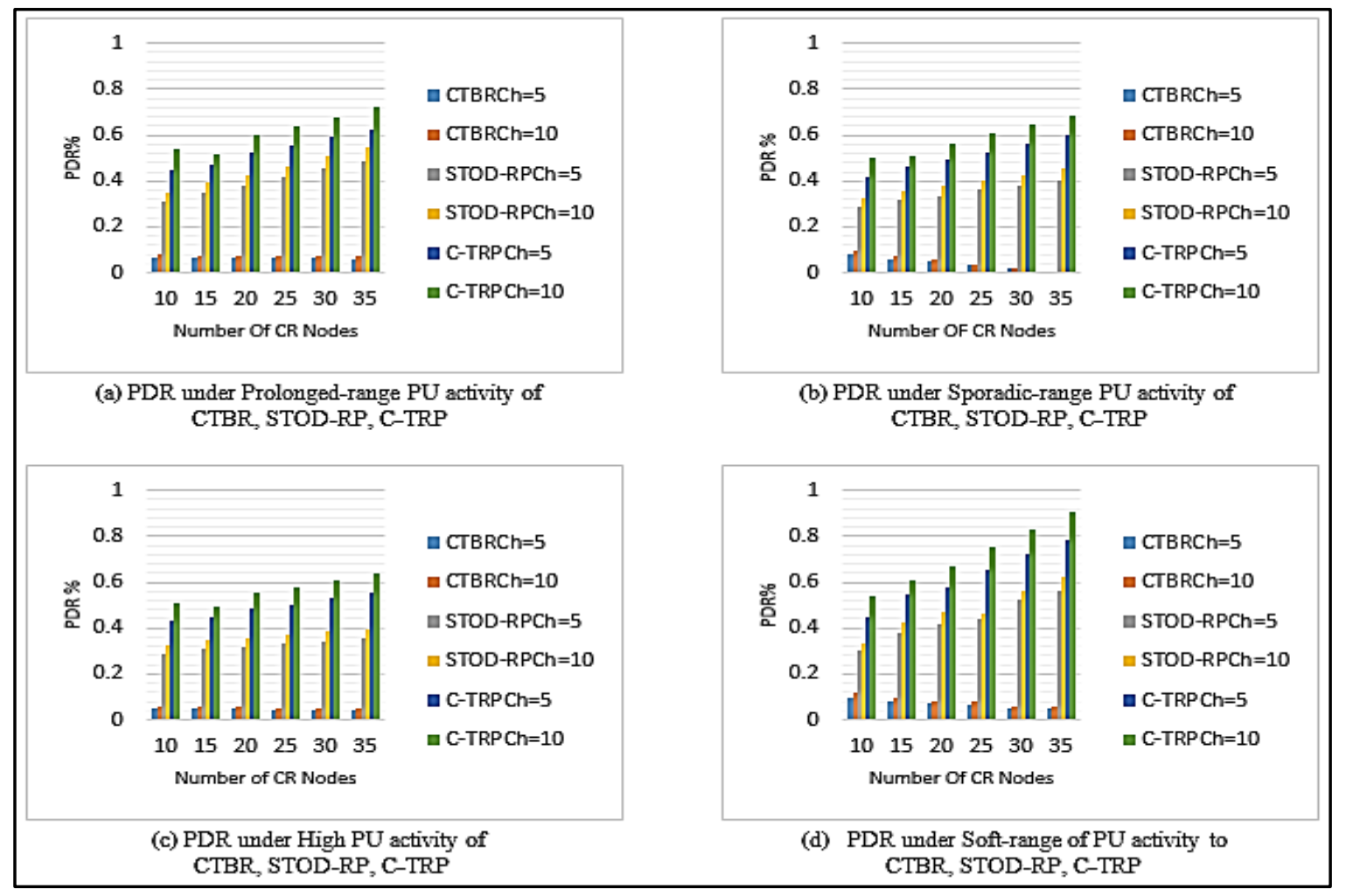

Figure 4 Comparison of PDR among CTBR, STOD-RP, C-TRP Under impact of different PU Activity patterns and different Number of Channels

Figure 4(a-d) evaluates the PDR under impact of differentPUs' activity patterns .PDR is affected also by the PUs activity duration. As PU remains more idle the PDR delivery increase as figure3(d).CTRP give better PDR in low PR activity pattern and lowest PDR in high user activity pattern.PDR significantly increases when the $P_{O F F}(t)$ increase.

\subsubsection{End-to-End Delay}

In this section comparing the performance of the three routing protocols in terms of delay from end to end. The comparison is done by changing the number of channels in the Free State and measuring the end-to-end delays based on random hops ranging from 2 to 10 nodes. Both the CTBR and STODRP protocols are not considering of the delay parameter when choosing the transmission free channel.The nodes select a random channel if the channel is not available, they will wait until the beginning of the next time slot to sense a random channel again. In our proposed protocol, channels are selected according to the availability of the channel and after that order the available channels according to the channels with the lowest delay. Note that before each transmission, the sender needs 
to check the selected channel to ensure it is free from PUs activity. Figure 5(a-d) shows the average end to end delay. The data flows are generated at random source SUs nodes from 2 to 10 hops away from the sink. The delay of each packet is measured from the time when it is generated at the source until it is received by the sink.

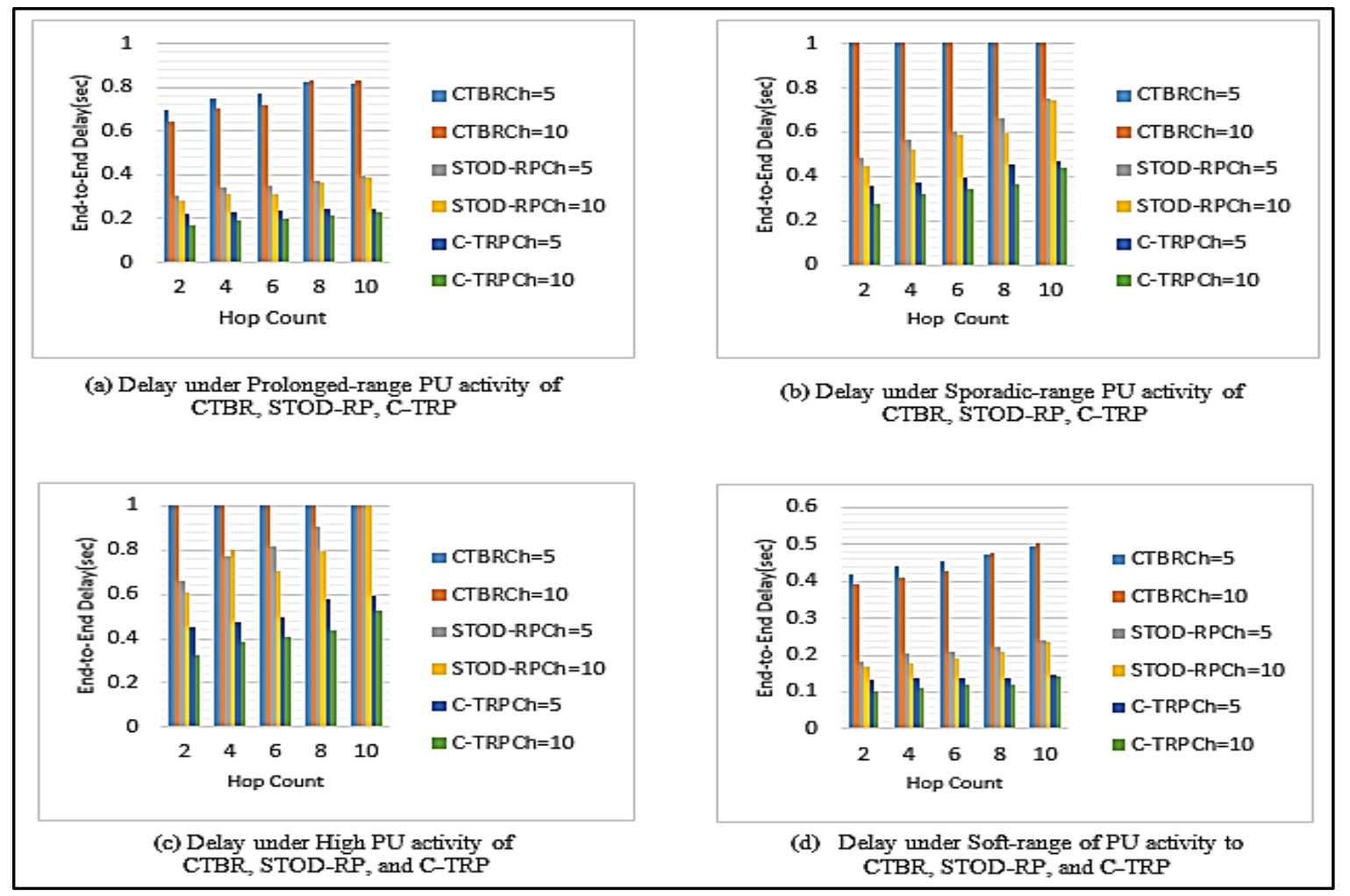

Figure 5 Comparison of End-to-End delay among CTBR, STOD-RP, C-TRPUnder impact of different PR Activity patterns and different Number of Channels

Figure 5(a-d) demonstrates that the delay is increased as the number of hops is increased. Because, calculating the delay by the sum of all delays at each SUs node from the source to the sink. Channel delay is decreasing when we increase the channels available from 5 to 10.This will increase the probability of SUs nodes to find available channels so the queuing packets at each node will decrease. The switching delay will also decrease because the node doesn't need to switch to another channel according to the probability of available channel time increase. All approaches achieve a better delay performance when $P_{O F F}(t)$ became longer. As our expectations, the end-to-end delay is increase when High-RangePUs activity is applied and improve in Sporadic-Range and ProlongedRange patterns and give the better delay performance in Soft-RangePUs pattern. 


\section{Conclusion}

In this paper, we have proposed a tree routing protocol for cognitive radio network (C-TRP) to improve the route selection decision in CRN. The different four patterns of PUs activity and the impact of each pattern on the performance of the three routing protocols (CTBR, STOD-RP and CTRP) was studied,we performed an extensive NS-2 simulations. The conclusion that routing protocols are most affected by the PUs activities and the number of channels available. More particularly our routing protocolC-TRP outperformed CTBR, STOD-RP in terms of three metrics: PDR, less interference to PUs nodes and also less end to end delay in multi-hop transmission under different of PUs' activities patterns.

\section{References}

1. McHenry, M., 2003. Spectrum White Space Measurements. Presentation to New America Foundation's Broadband Forum. June 20, pp: 1-13. Retrieved from: http://www.newamerica.net/files/nafmigration /archive/Doc_File_185_1.pdf

2. FCC's Spectrum Policy Task Force, 2002. Report of the spectrum efficiency working group. Technical Report, Federal Communications Commission (FCC). Retrieved from: http://transition.fcc.gov/sptf/files/SEWGFinalReport_1.pdf.

3. Akyildiz, I.F., W.Y. Lee, M.C. Vuran and S. Mohanty, 2006. Next generation/dynamic spectrum access/cognitive radio wireless networks: A survey. Comput. Netw., 50(13): 2127-2159.

4. Haykin, S., 2005. Cognitive radio: Brain-empowered wireless communications. IEEE J. Sel. Area Comm., 23(2): 201-220.

5. Mitola, J., 2009. Cognitive radio architecture evolution. P. IEEE, 97(4): 626-641.

6. Akyildiz, I.F., W.Y. Lee and K.R. Chowdhury, 2009. CRAHNs: Cognitive radio ad hoc networks. Ad Hoc Netw., 7(5): 810-836.

7. Marinho, J. and E. Monteiro, 2012. Cognitive radio: Survey on communication protocols, spectrum decision issues and future research directions. Wirel. Netw., 18(2): 147-164.

8. Cesana, M., F. Cuomo and E. Ekici, 2011. Routing in cognitive radio networks: Challenges and solutions. Ad Hoc Netw., 9(3): 228-248.

9. Sengupta, S. and K.P. Subbalakshmi, 2013. Open research issues in multi-hop cognitive radio networks. IEEE Commun. Mag., 51(4): 168-176.

10. Zhu G, Akyildiz IF, Kuo G. STOD: a spectrum-tree based on demand routing protocol for multihop cognitive radio networks. In: Proc IEEE Globecom. p.1-5.

11. Zhang B, Takizawa Y, Hasagawa A, Yamauchi A, Obana S. Tree-based routing protocol for cognitive wireless access networks. In: Proc of IEEE WCNC 2007, March 2007. p. 4207-11.

12. M. Hashem, S. I. Barakat, M. A. AttaAlla , "A tree routing protocol for cognitive radio network", Egyptian Informatics Journal- 10 November 2016. [online] Available: https://doi.org/10.1016/j.eij.2016.10.001

13. Rocha, F., 2012. NS2 Visual Trace Analyzer. Retrieved from:http://nsvisualtraceanalyzer. wordpress.com/.

14. W.-Y. Lee and I. Akyildiz, "Optimal spectrum sensing framework for cognitive radio networks," IEEE Transactions on Wireless Communications, vol. 7, no. 10, pp. 3845 -3857, october 2008.

15. G. Yuan, R. Grammenos, Y. Yang, and W. Wang, "Performance analysis of selective opportunistic spectrum access with traffic prediction," IEEE Transactions on Vehicular Technology, vol. 59, no. 4, pp. $1949-1959$, may 2010. 
16. W. Min and K. G. Shin, "Exploiting multi-channel diversity in spectrum-agile networks," in Proceedings of INFOCOM, 13-18 April 2008, pp. 1921 - 1929.

17. M. Di Felice, K. R. Chowdhury, and L. Bononi, "Modeling and performance evaluation of transmission control protocol over cognitive radio ad hoc networks," in Proceedings of the 12th ACM international conference on Modeling, analysis and simulation of wireless and mobile systems, ser. MSWiM '09. New York, NY, USA: ACM, 2009, pp. 4-12. [Online]. Available: http://doi.acm.org/10.1145/1641804.1641809.

18. S. Bayhan and F. Alags, "Distributed channel selection in crahns: A non-selfish scheme for mitigating spectrum fragmentation," Ad Hoc Networks, vol. In Press, Corrected Proof, pp. -, 2011. [Online]. Available: http://www.sciencedirect.com/ science/article /pii/S1570870511000941

19. J. Riihijarvi, J. Nasreddine, and P. Mahonen, "Impact of primary user activity patterns on spatial spectrum reuse opportunities," in European Wireless Conference (EW 2010), 2010.

20. M. Hashem, S. I. Barakat, M. A. AttaAlla, "DCSS:Distributed Channel Selection Strategy Based On Channel Weight In Multi-Hop Cognitive Radio Network", International Journal of Intelligent Computing and Information Sciences (IJICIS), Vol.16 No. 2 APRIL 2016. 